\title{
Runtime vs Scheduler: Analyzing Dask's Overheads
}

\author{
Stanislav Böhm \\ IT4Innovations, VSB - Technical University of Ostrava \\ stanislav.bohm@vsb.cz
}

\author{
Jakub Beránek \\ IT4Innovations, VSB - Technical University of Ostrava \\ jakub.beranek@vsb.cz
}

\begin{abstract}
Dask is a distributed task framework which is commonly used by data scientists to parallelize Python code on computing clusters with little programming effort. It uses a sophisticated work-stealing scheduler which has been handtuned to execute task graphs as efficiently as possible. But is scheduler optimization a worthwhile effort for Dask? Our paper shows on many real world task graphs that even a completely random scheduler is surprisingly competitive with its built-in scheduler and that the main bottleneck of Dask lies in its runtime overhead. We develop a drop-in replacement for the Dask central server written in Rust which is backwards compatible with existing Dask programs. Thanks to its efficient runtime, our server implementation is able to scale up to larger clusters than Dask and consistently outperforms it on a variety of task graphs, despite the fact that it uses a simpler scheduling algorithm.
\end{abstract}

Index Terms-distributed task scheduling, dask, workflow, rust

\section{INTRODUCTION}

Distributed task frameworks are commonly used to scale programs to multiple nodes using little programming effort. While traditional HPC distributed paradigms like MPI promote fixed-size clusters with little resilience and low-level communication APIs designed for maximum performance, modern task frameworks like DASK [1], Ray [2] or Spark [3] support elastic clusters and provide high-level programming interfaces which abstract the communication aspect of the program away. They offer quick prototyping, even though they do not always provide the highest possible performance out-of-the-box. These frameworks are especially popular for distributing machine learning and data analysis programs with a few lines of code.

Even though each task framework offers its own set of APIs that enable writing distributed programs, they all eventually convert the input program into a task graph. Vertices of this graph (called tasks) represent functions which operate on input data and generate output data. Arcs represent dependencies and data transfers between the tasks. This program representation is amenable to parallelization in a distributed environment. The job of a task framework is to decide on which computing

This work was supported by The Ministry of Education, Youth and Sports from the National Programme of Sustainability (NPS II) project "IT4Innovations excellence in science - LQ1602" and by The Ministry of Education, Youth and Sports from the Large Infrastructures for Research, Experimental Development, and Innovations project "e-INFRA CZ - LM2018140". This work was also partially supported by the SGC grant No. SP2020/167 "Extension of HPC platforms for executing scientific pipelines 2", VŠB - Technical University of Ostrava, Czech Republic. nodes should the individual tasks be computed and manage data transfers between the nodes.

A crucial component of each task framework is the scheduler, which assigns tasks to nodes in order to minimize the total computation time. Finding the optimal task schedule is a wellknown problem which is NP-hard even for very restricted formulations (e.g. even without network data transfers) [4]. A number of heuristics have been proposed to tackle this problem, ranging from list-based scheduling to genetic algorithms [5][11]. Many surveys and comparisons of these approaches were published in [12] $-[15]$. Yet applying these algorithms to modern task frameworks is challenging, as they often make assumptions that do not hold in practice. For example they often assume that task durations are known in advance or that there is no network congestion. Task frameworks thus usually resolve to implementing their own scheduler with many heuristic decisions specific to their common use cases.

One example of such a framework is DASK, a popular Python library used to distribute and parallelize SQL-like table operations, machine learning workflows or even arbitrary Python functions with little programming effort. DASK uses a centralized architecture with a single central component the server - which schedules tasks and manages computing nodes in a cluster. It uses a work-stealing scheduler which has been tuned extensively to support various task graphs. Yet it is unclear whether additional effort should be directed into improving the scheduler or if there is another bottleneck which should be prioritized.

We have designed a series of experiments that study the runtime overhead of DASK and the effect of the used scheduling algorithm on its performance. We also develop a backwards compatible replacement of the DASK server which aims to minimize its runtime overhead and thus scale to larger clusters. Our paper makes the following contributions:

- We demonstrate that even a very naïve scheduling algorithm, such as a completely random scheduler, is in many common scenarios competitive with the sophisticated hand-tuned work-stealing scheduler used by DASK.

- We develop RSDS, an open-source drop-in replacement for the DASK server compatible with existing DASK programs that consistently outperforms the DASK server in a diverse benchmark set, which we also openly released.

- We quantify and evaluate DASK's task overhead using an idealized worker implementation on various task graphs. 
The structure of the paper is as follows. First we describe the architecture of DASK in section III and the specifics of our server reimplementation in Rust [16] in section IV. We have designed several experiments to study the performance limits of DASK and compare its performance with RSDS. Our benchmarks are described in Section $\mathrm{V}$ and the evaluation is in Section VI Lastly we conclude our findings in Section VII

\section{RELATED WORK}

A number of task framework benchmarks that included DASK have been published. The author of DASK has performed a series of DASK scaling microbenchmarks [17]. In [18], the authors benchmark several distributed task systems on a set of fundamental task graph shapes to compare their relative overhead and scaling properties. They find out that DASK stops scaling relatively quickly if the task granularity is smaller than ca. one hundred milliseconds, which we also confirm in our experiments. The performance of DASK vs Spark on a neuroimaging pipeline is compared in [19]. Their results suggest that multi-threaded DASK workers have limited usability, which we also confirm in our evaluation. In [20], a data science benchmark is performed that compares the performance of common Python, SQL, and R data analysis libraries on various task graphs. A task scheduler and a distributed runtime was developed in [21]. It compares its performance to DASK on one real-world and two synthetic task graphs. It is not backwards compatible with DASK.

None of the mentioned works examine the effect of the used scheduling algorithm in DASK nor do they implement minimal overhead baselines for the DASK server and worker.

\section{DASK}

DASK is a flexible Python library for parallel computation in Python. It offers APIs compatible with popular Python libraries like pandas ${ }^{1}$ for table processing or NumPy for n-dimensional array operations. Code that uses these interfaces is automatically transformed into a task graph, which is then executed in parallel. This enables almost transparent parallelization of sequentially looking Python code. Apart from these high-level interfaces, it is also possible to build the task graph manually from scratch.

\section{A. Task graph}

The core representation of a distributed program in DASK is the task graph. It is a directed acyclic graph (DAG), where the vertices (tasks) are Python functions that should be computed and arcs are dependencies between tasks. An arc from a task $u$ to a task $v$ represents that task $v$ needs to use the output produced by task $u$ as one of its input parameters. Therefore $u$ has to be finished before $v$ can begin executing and the output of $u$ has to be transferred over the network if $v$ is scheduled to a different computing node than $u$.

${ }^{1}$ https://pandas.pydata.org

${ }^{2}$ http://numpy.org

\section{B. Architecture}

DASK supports multiple computing backends. In this work we focus solely on Dask/distributed 3 , which computes task graphs on a distributed cluster. When we refer to DASK, we assume that it uses this distributed backend. Its architecture is composed of three main components: the client, the server and the worker.

Client is the user-facing API used to run distributed computations. The client code connects to a DASK cluster, submits task graphs to the server and gathers the results.

Server is the central component of the DASK cluster which coordinates workers and handles requests from clients. It contains a scheduler which assigns tasks to workers to load balance the cluster. Each DASK cluster has a single server.

Worker is a process which executes tasks (Python functions) submitted by the client. Server sends tasks (consisting of serialized Python functions and arguments) to individual workers to be executed. Workers communicate amongst themselves to exchange task outputs that are not available locally. Each worker is configured with a number of CPU cores that it is allowed to use. Workers process their tasks in parallel, but they never execute more than one task per available core at once.

\section{Programming interface}

DASK allows defining computational workflows in several ways. At the lowest level is the Futures interface which can lazily build a task graph from Python functions. On top of Futures DASK offers high-level interfaces that mimic the API of popular Python libraries. Examples of these include Arrays, a parallel version of the NumPy library for numerical computations on tensors or DataFrame, a parallel version of the Pandas library for analysis of tabular data.

These interfaces use the concept of partitions. It is a parameter which controls the granularity of tasks created by DASK. For example, an operation on a large table might be partitioned into several tasks, each operating on a subset of rows. The selected amount of partitions specifies into how many parts will the table be divided. It is crucial to choose this parameter accurately, otherwise the task graph might not be parallelized effectively.

\section{Work-stealing scheduler}

DASK uses a sophisticated work-stealing scheduler containing many heuristics that have been tuned for quite some time. Some of them are described in the DASK manual 4 The scheduler works in the following way: when a task becomes ready, i.e. all its dependencies are finished, it is immediately assigned to a worker according to a heuristic that tries to minimize an estimated start time of the task. The estimate is based on potential data transfers and the current occupancy of workers. When an imbalance occurs (some workers are underloaded/overloaded), the scheduler tries to steal tasks from overloaded nodes and distribute them to underloaded nodes.

3 https://distributed.dask.org

${ }^{4}$ https://distributed.dask.org/en/latest/work-stealing.html 
The scheduler also assigns priorities to tasks that are used by workers to decide what tasks should be computed first.

\section{E. Integrating schedulers into DASK}

Integrating existing task scheduling algorithms into DASK is difficult, because the work-stealing concept is integrated tightly inside DASK. Integrating an existing algorithm would thus require making modifications both to DASK and to the algorithm via heuristic decisions which could introduce bias.

To avoid that, we have implemented possibly the simplest possible scheduling algorithm - a random scheduler. A completely random scheduler does not need any additional heuristics and is therefore immune from any possible bias that we could introduce by our implementation. It is also so simple that it could be included in DASK without making large modifications to it. Our random scheduler eagerly assigns each task to a random worker using a uniform random distribution.

\section{REIMPLEMENTING THE DASK SERVER}

To examine the runtime overhead of DASK, we needed a baseline for the server implementation with the lowest possible overhead. To get close to this goal, we have reimplemented the server in Rust. Rust has a minimal runtime and manual memory management, which by itself reduces the ubiquitous overhead of reference counting and indirection present in Python. It also has direct programming support for asynchronous I/O and it provides strong memory safety guarantees, hence it is well suited for writing distributed applications. We call our server reimplementation RSDS.

RSDS is compatible with existing DASK clients and workers and it can thus be used to run DASK programs without making any source code changes. Even though it is not feature complete, it supports a minimum set of DASK message types which are necessary to run the most common DASK workflows. RSDS is able to run a diverse set of unmodified DASK programs described in Section V. We provide RSDS as open-source software at https://github.com/it4innovations/rsds

\section{A. Architecture}

The architecture of RSDS is shown in Figure 11. The main architectural difference between RSDS and DASK is the separation of the server into two parts: reactor and scheduler. The reactor manages worker and client connections, maintains bookkeeping information and translates scheduler assignments into DASK messages which are then sent to the workers.

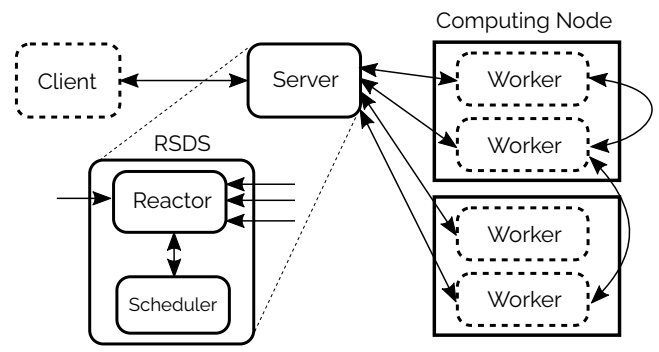

Fig. 1. Architecture of RSDS (Dask components are dashed)
The scheduler is a process which receives a task graph and outputs assignments of tasks to workers. It does not care about network connections, the DASK protocol messages or any other bookkeeping that is not relevant to scheduling. Since it is isolated, it can be swapped easily and therefore it is trivial to experiment with different schedulers in RSDS. Another benefit is that we can easily run the scheduler in a separate thread to enable concurrent scheduling and runtime management. This is possible because the scheduler does not share any data structures with the reactor. A disadvantage of this scheme is that the both the reactor and the scheduler need to build their own task graph, which increases memory usage, but we have not found this to be a problem in practice.

\section{B. DASK protocol modifications}

DASK uses a custom language-agnostic communication protocol serialized by MessagePack 5 DASK sometimes arbitratily fragments message structures into parts and then reassembles them during deserialization. This is difficult to perform in a statically typed language. We have modified it to keep the original message structure and replace values that must be fragmented with placeholders, which are later replaced with deserialized values. This avoids the need to dynamically change the message structure during deserialization.

Our change only modifies low-level message handling, it is thus fully transparent to the rest of the code and spans less than 100 modified lines of DASK source code. It has no effect on the functionality of clients, workers and the server. Our modified version of DASK is open-source and available online ${ }^{6}$ All evaluations presented in this paper use the modification described above, even when RSDS is not used. We have benchmarked this modification and found that there are no performance differences in respect to the original DASK message encoding.

\section{Schedulers}

We have implemented two schedulers in RSDS - a workstealing scheduler and a random scheduler - to test the runtime overhead difference between DASK and RSDS while using a similar set of schedulers.

Even though it was not possible to exactly replicate the workstealing implementation used in DASK, our implementation is inspired by it. However, it is also deliberately simple to avoid the need to perform extensive hand-tuning. Some of the heuristics used by DASK were changed, simplified, or dropped in our implementation. For example RSDS does not estimate average task durations and does not use any network speed estimates.

The RSDS work-stealing scheduler works as follows: when a task becomes ready (i.e. all its inputs are already finished), it is immediately assigned to a worker. The scheduler chooses a worker where the task may be executed with minimal data transfer costs, while it deliberately ignores the load of the worker. The load is ignored to speed up the decision in

5 https://msgpack.org

6 https://github.com/kobzol/distributed/tree/simplified-encoding 
optimistic situations when there is enough tasks to keep the workers busy. When it is not the case, it is solved by balancing (described below).

For computing transfer costs, we use a heuristic that takes into account inputs that are already present on the worker's node and also inputs that will be eventually present because they are in transit or they are depended upon by another task assigned to the same worker. Transfer cost is smaller for data transfers between workers residing on the same node.

When a new task is scheduled or when a task is finished, the scheduler checks if there are nodes that are under-loaded. In such case, balancing is performed and the scheduler reschedules tasks from workers with sufficient number of tasks to under-loaded workers. During rescheduling, the scheduler simply passes the new scheduling state to the reactor, which performs all of the complex rescheduling logic. It tries to retract rescheduled tasks from their originally assigned workers. If retraction succeeds, the task is scheduled to the newly assigned worker. When the retraction fails, because a task is already running or has been finished, the scheduler is notified and it then initiates balancing again if necessary.

Our random scheduler mirrors the random scheduler implementation in DASK - it assigns a random worker using a uniform random distribution to each task as soon as the task arrives to the server. It ignores any other scheduling mechanisms, such as task stealing, and does not maintain any task graph state.

\section{Zero worker}

To quantify the runtime overhead of the DASK server, we have created an implementation of the DASK worker that we call zero worker. Zero worker is a minimal implementation of the DASK worker process written in Rust. Its purpose is to simulate a worker with infinite computational speed, infinitely fast worker-to-worker transfers and zero additional overhead. It actually does not perform any real computation; when a task is assigned to a zero worker, it immediately returns a message that the task was finished. It also remembers a set of data-objects that would be placed on the worker in a normal computation. When a task requires a data object which is not in this list, the worker immediately sends a message to the server that the object was placed on it - this simulates an infinitely fast download of data between workers. No actual worker-to-worker communication is performed in such case.

Zero workers respond to every data fetch request with a small mocked constant data object. Such requests come from the server when a client asks for a data object, usually at the end of the computation. Since there is no worker-to-worker communication, fetch requests never come from other workers.

We use the zero worker in our experiments only when explicitly stated; otherwise, we always use the original DASK worker implementation.

\section{BENCHMARKS}

This section covers task graphs benchmarked in our experiments. We have prepared a diverse set of benchmarks that span from simple map-reduce aggregations to text processing workloads and table queries. The properties of the task graphs used in our experiments along with the DASK API that was used to create them are summarized in Table I. Most of the task graphs are heavily inspired by programs from the DASK Examples repository 7 . Our benchmark dataset is available in the RSDS repository. A short summary of the individual benchmarks is provided below.

merge-n creates $n$ independent trivial tasks that are merged at the end. This benchmark is designed to stress the scheduler and the server.

merge_slow-n-t is similar to merge-n, but with longer, $t$ second tasks.

tree-n performs a tree reduction of $2^{n}$ numbers using a binary tree with height $n-1$.

xarray-n calculates aggregations (mean, sum) on a threedimensional grid of air temperatures [22], $n$ specifies size of grid partitions.

bag-n-p works with a dataset of $n$ records in $p$ partitions. It performs a cartesian product, filtering and aggregations.

numpy-n-p transposes and aggregates a two-dimensional distributed NumPy array using the Arrays interface. The array has size $(n, n)$ and it is split into partitions of size $(n / p, n / p)$. groupby-d-f-p works with a table with $d$ days of records, each record is $f$ time units apart, records are partitioned by $p$ time units. It performs a groupby operation with an aggregation.

join-d-f-p uses the same table, but performs a self-join.

vectorizer-n-p uses Wordbatch ${ }^{8}$, a text processing library, to compute hashed features of $n$ reviews from a TripAdvisor dataset [23] split into $p$ partitions.

wordbag-n-p uses the same dataset, but computes a full text processing pipeline with text normalization, spelling correction, word counting and feature extraction.

\section{EXPERIMENT EVALUATION}

This section presents our evaluation setup and methodology and discusses the results of several experiments that we have designed to test DASK schedulers and compare our RSDS server with the original DASK server implementation.

All experiments were performed on the Salomon supercomputer 9 . Each Salomon node has two sockets containing Intel Xeon E5-2680v3 with 12 cores clocked at $2.5 \mathrm{GHz}$ (24 cores in total), $128 \mathrm{GiB}$ of RAM clocked at $2133 \mathrm{MHz}$ and no local disk. The interconnections between nodes use InfiniBand FDR56 with 7D enhanced hypercube topology.

In all of our experiments, we use a setting with 24 DASK workers per node, each using a single thread for task computations. We chose this setting because of the Global Interpreter Lock present in the standard CPython interpreter. Since our benchmarks are compute-bound and not I/O-bound, a single worker cannot effectively use more than a single thread. Not even the popular NumPy and Pandas libraries used in our benchmarks are multi-threaded by default, which is also why

'https://examples.dask.org

$\varepsilon^{\text {https://github.com/antttti/Wordbatch }}$

' https://docs.it4i.cz/salomon/introduction 
TABLE I

TASK GRAPH PROPERTIES

\begin{tabular}{l|rrrrrc}
\hline Task graph & \#T & \#I & S & AD & LP & API \\
\hline merge-10K & 10001 & 10000 & 0.027 & 0.006 & 1 & $\mathrm{~F}$ \\
merge-15K & 15001 & 15000 & 0.027 & 0.006 & 1 & $\mathrm{~F}$ \\
merge-20K & 20001 & 20000 & 0.027 & 0.006 & 1 & $\mathrm{~F}$ \\
merge-25K & 25001 & 25000 & 0.027 & 0.006 & 1 & $\mathrm{~F}$ \\
merge-30K & 30001 & 30000 & 0.027 & 0.006 & 1 & $\mathrm{~F}$ \\
merge-50K & 50001 & 50000 & 0.027 & 0.006 & 1 & $\mathrm{~F}$ \\
merge-100K & 100001 & 100000 & 0.027 & 0.006 & 1 & $\mathrm{~F}$ \\
merge_slow-5K-0.1 & 5001 & 5000 & 0.023 & 100 & 1 & $\mathrm{~F}$ \\
merge_slow-20K-0.1 & 20001 & 20000 & 0.023 & 100 & 1 & $\mathrm{~F}$ \\
tree-15 & 32767 & 32766 & 0.027 & 0.007 & 14 & $\mathrm{~F}$ \\
xarray-25 & 552 & 862 & 55.7 & 3.1 & 10 & $\mathrm{X}$ \\
xarray-5 & 9258 & 14976 & 3.3 & 0.4 & 10 & $\mathrm{X}$ \\
bag-25K-10 & 236 & 415 & 292 & 1233 & 6 & $\mathrm{~B}$ \\
bag-25K-100 & 21631 & 41430 & 3.2 & 13.9 & 8 & $\mathrm{~B}$ \\
bag-25K-200 & 86116 & 165715 & 0.8 & 3.6 & 9 & $\mathrm{~B}$ \\
bag-25K-50 & 5458 & 10357 & 12.6 & 54.9 & 7 & $\mathrm{~B}$ \\
bag-50K-50 & 5458 & 10357 & 25.2 & 214 & 7 & $\mathrm{~B}$ \\
numpy-50K-10 & 209 & 228 & 70108 & 169 & 7 & $\mathrm{~A}$ \\
numpy-50K-100 & 19334 & 21783 & 760 & 2.6 & 10 & $\mathrm{~A}$ \\
numpy-50K-200 & 77067 & 86966 & 191 & 0.9 & 11 & $\mathrm{~A}$ \\
numpy-50K-50 & 4892 & 5491 & 2999 & 8.3 & 9 & $\mathrm{~A}$ \\
groupby-2880-1S-16H & 22842 & 31481 & 1005 & 11.9 & 9 & $\mathrm{D}$ \\
groupby-2880-1S-8H & 45674 & 62953 & 503 & 7.7 & 9 & $\mathrm{D}$ \\
groupby-1440-1S-1H & 182682 & 251801 & 64.3 & 3.8 & 10 & $\mathrm{D}$ \\
groupby-1440-1S-8H & 22842 & 31481 & 503 & 7.7 & 9 & $\mathrm{D}$ \\
groupby-360-1S-1H & 45674 & 62953 & 64.3 & 3.8 & 9 & $\mathrm{D}$ \\
groupby-360-1S-8H & 5714 & 7873 & 503 & 8.0 & 8 & $\mathrm{D}$ \\
groupby-90-1S-1H & 11424 & 15743 & 64.3 & 3.9 & 8 & $\mathrm{D}$ \\
groupby-90-1S-8H & 1434 & 1973 & 501 & 7.7 & 7 & $\mathrm{D}$ \\
join-1-1S-1H & 673 & 1224 & 15.3 & 33.0 & 5 & $\mathrm{D}$ \\
join-1-1S-1T & 72001 & 125568 & 3.7 & 1.7 & 11 & $\mathrm{D}$ \\
join-1-2s-1H & 673 & 1224 & 9.3 & 9.8 & 5 & $\mathrm{D}$ \\
vectorizer-1M-300 & 301 & 0 & 10226 & 1504 & 0 & $\mathrm{~F}$ \\
wordbag-100K-50 & 250 & 200 & 5136 & 301 & 2 & $\mathrm{~F}$ \\
\hline & & & & & & \\
\hline
\end{tabular}

$\# \mathrm{~T}=$ Number of tasks; \#I = Number of dependencies;

$\mathrm{S}=$ Average task output size $[\mathrm{KiB}] ; \mathrm{AD}=$ Average task duration $[\mathrm{ms}]$; $\mathrm{LP}=$ longest oriented path in the graph;

$\mathrm{D}=$ DataFrame $; \mathrm{B}=\mathrm{Bag} ; \mathrm{A}=$ Arrays; $\mathrm{F}=$ Futures; $\mathrm{X}=$ XArray

DASK provides direct API support for their parallelization. To confirm our decision, we have benchmarked a configuration using a single worker with 24 threads per each node. We have found that it provides no benefit in comparison to a single worker with only one thread in any of our benchmarks. These tests are not reported in the paper. The fact that some workflows do not benefit from multi-threaded DASK workers has been also observed in [19].

For each of our experiments, we state the number of used worker nodes, these contain only the workers. We always use one additional node which runs both the client and the server. For our scaling experiments we use 1 to 63 worker nodes (241512 DASK workers), for the rest of our experiments we use either 1 or 7 worker nodes (24 or 168 DASK workers). We have chosen these two cluster sizes to represent a small and a medium sized DASK cluster. The number of workers is fixed, they do not connect nor disconnect during the computation. The timeout for all benchmarks was set to 300 seconds.

We have run each benchmark configuration five times (except for the scaling benchmarks, which were executed two times to lower our computational budget) and averaged the

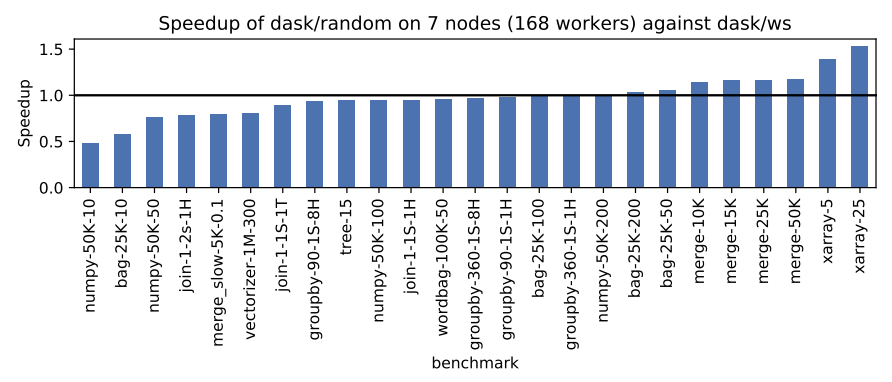

Fig. 2. Speedup of DASK/random scheduler; DASK/ws is baseline.

result. We measure the duration between the initial task graph submission to the server and the processing of the final output task by the client. We call this duration makespan. We reset the whole cluster between each benchmark execution.

We use the following abbreviations in figures with benchmark results: $w s$ marks the work-stealing scheduler and random represents the random scheduler.

\section{A. Scheduler}

Our first experiment compares the built-in work-stealing scheduler vs a random scheduler used in the DASK server. The overall results are shown in Figure 2. To our surprise, we have found out that the simple random scheduler fares relatively well on both small and medium sized clusters. At worst, it produces a twice longer makespan, but overall it is quite close to the performance of the work-stealing scheduler and in some cases it even outperforms it with a $1.4 \times$ speedup. The geometric mean of speedup in comparison to the workstealing scheduler over all tested task graphs can be observed in Table II It can be seen that the random scheduler gets closer to the performance of work-stealing if more workers are used.

There are two reasons for this. Firstly, with more workers, the work-stealing scheduler has to do more work to compute where should the tasks be computed and it also generates additional network traffic by sending task stealing messages. The random scheduler has a fixed computation cost per task independent of the worker count. It simply chooses a worker randomly and it sends no additional messages other than one assignment per task. The second reason is that our benchmark set is more computationally bounded rather than network bounded. Therefore, with more workers, if the scheduler wants to utilize all computational power of the cluster, network transfers are less avoidable. This decreases the chance that a random scheduler induces an unnecessary data transfer that could have been avoided by a smarter scheduler.

The results of this experiment suggest that in many scenarios, a complex scheduling algorithm is not needed and a random schedule is sufficient for DASK. This opens up the question whether the schedule is the main bottleneck which slows down the execution of DASK workflows. We try to answer it in the following experiments. 


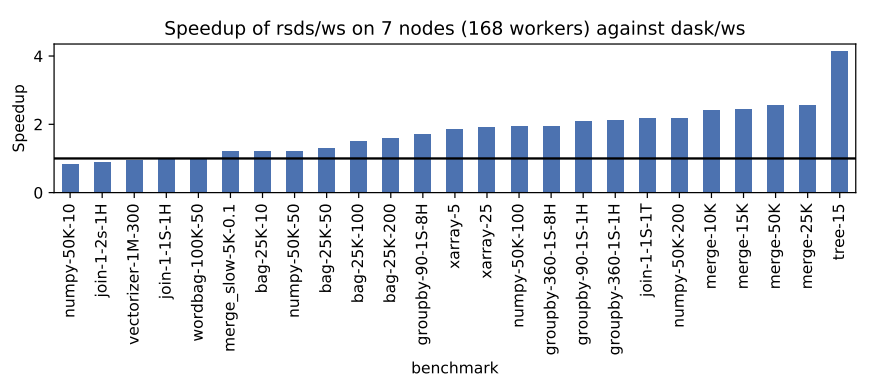

Fig. 3. Speedup of RSDS/ws scheduler; DASK/ws is baseline.

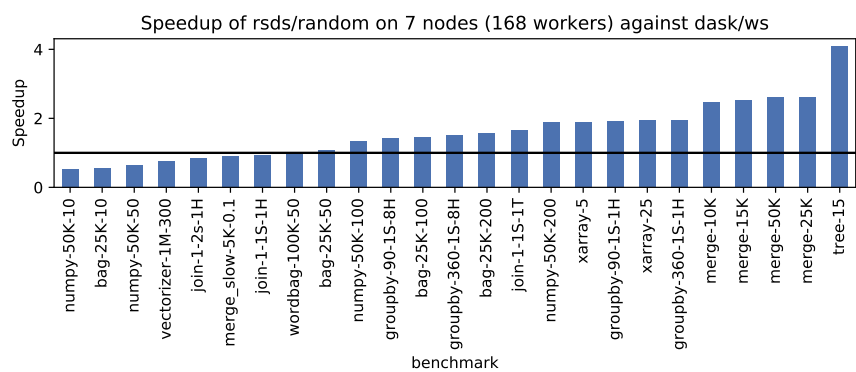

Fig. 4. Speed up RSDS/random scheduler; DASK work-stealing is baseline.

\section{B. Server}

This experiment compares the efficiency of RSDS and the original DASK server with both the work-stealing and the random scheduler. Results for work-stealing schedulers are shown in Figure 3. The data confirm our expectation that DASK has large inherent overhead and that reducing it helps to improve the makespan of executed task graphs. Even though RSDS uses a much simpler work-stealing scheduler, its more efficient runtime provides better performance in most cases and this effect becomes even more visible with larger clusters. Figure 4 confirms that the speedup is caused by the reduced runtime overhead and not by the different workstealing implementation. It shows the speedup of RSDS using a random scheduler over DASK with the work-stealing scheduler. This serves as an evidence that the improved performance of RSDS with work-stealing is caused by better runtime efficiency and not by better schedules.

TABLE II

GEOMETRIC MEAN OF SPEEDUP FOR EXPERIMENTS A (SCHEDUleR) AND B (SERVER); BASELINE IS DASK/WS

\begin{tabular}{c|c|r|r|r} 
Server & Scheduler & Node count & Worker count & Speedup \\
\hline dask & random & 1 & 24 & $0.88 \times$ \\
dask & random & 7 & 168 & $0.95 \times$ \\
rsds & random & 1 & 24 & $1.04 \times$ \\
rsds & random & 7 & 168 & $1.41 \times$ \\
rsds & ws & 1 & 24 & $1.28 \times$ \\
rsds & ws & 7 & 168 & $1.66 \times$
\end{tabular}

\section{Scaling}

To examine how DASK scales to larger clusters and whether the reduced overhead of RSDS can improve scaling, we have designed an experiment which tests the strong scaling of both servers on several cluster sizes ranging from 1 ( 24 workers) to 63 nodes (1512 workers). The results of this experiment are shown in Figure 5. The work-stealing algorithm was used for both server implementations.

The first examined task graph is merge-100K, which executes hundred thousand trivially short tasks. It is an adversarial case for a scheduler, as the tasks are short and thus the overhead of scheduling and network transfers will overcome most parallelism gains. Therefore, increasing the amount of workers should not provide a large speedup for this task graph. However, it should ideally not slow down the computation to a large extent. We can see that RSDS scales only up to 15 nodes (360 workers). This is caused by the fact that the cost associated with worker management and workstealing raises with an increasing number of workers and from some point it starts to dominate because the tasks are too short. DASK is twice slower when compared to RSDS with a single worker node and it is four times slower on 63 nodes (1512 workers). Here we can see that the inner overhead of DASK adds up and its performance is reduced significantly with each additional worker node.

Next we examine groupby-2880-1S-16H, which computes an analysis of table data using a task graph automatically generated by DASK by the pandas API. This task graph provides opportunities for parallelization, as the individual tasks work on a subset of rows and thus have more computational density compared to the merge task graph. However, Table I shows that the average computation time is still only around $10 \mathrm{~ms}$ while the average task output is $1 \mathrm{MiB}$. It thus produces considerable network traffic. While both DASK and RSDS have identical performance with a single worker node, DASK stops scaling at 7 nodes and further its performance degrades and eventually becomes slower than the single node case. RSDS scales up to 23 worker nodes, hence it is able to utilize three times more workers. With more worker nodes the performance of RSDS also degrades, as the network communication caused by task output transfers and work-stealing messages starts to dominate the overall execution time.

The third examined task graph is merge_slow-20K, which executes twenty thousand tasks where each task has a fixed duration specified by a parameter. Note that merge and merge_slow have the exact same task graph shape. The only difference is the duration of each task. We have benchmarked three variants of this task graph, with $0.01,0.1$ and 1 second tasks. This gives us a better idea of the task granularity required for DASK and RSDS to scale effectively. With 10 millisecond tasks, DASK scales to 7 workers and then its performance follows a similar shape as for merge-100K. RSDS stops scaling at 15 nodes, then its performance drops slightly with more added nodes. With 100 millisecond tasks, RSDS is able to scale up to 47 worker nodes (1128 workers), from that 
point on its performance stagnates. DASK scales only up to 23 worker nodes, then the makespan again starts to increase when additional workers are added. For the last task graph with one second tasks, both RSDS and DASK scale up to 63 nodes (1512 workers); however, RSDS is consistently faster on all cluster sizes and its performance in respect to DASK increases with added worker nodes; it is $1.03 \times$ faster than DASK with 7 nodes and $1.6 \times$ faster with 63 nodes.

This shows that for embarrassingly parallel task graphs, DASK needs at least ca. 100 millisecond task duration in order to scale up to a larger number of workers. Otherwise it will slow down with each added worker node. The reduced overhead of RSDS enables it to scale better and it also keeps its performance relatively stable with an increasing number of workers, even for short tasks.

\section{Worker overhead isolation}

We have seen that there is considerable overhead in the DASK server and that lowering that overhead can shorten makespans of executed task graphs. However, it is possible that other overheads in the DASK runtime might conceal additional inefficiency of the server. We test both the DASK server and RSDS with the zero worker - a minimal worker implementation that does not perform any real computation. Using an idealized worker implementation helps us understand the server performance better, because it then remains as the only bottleneck that could hinder the execution.

In this experiment, all benchmarks are executed with the zero worker. We use only a subset of our benchmark set in this experiment, as some of the benchmarks depend on concrete outputs of tasks and thus cannot be used with the zero worker which produces mock data. Note that since all of the tasks are computed immediately and have the same output size, the only difference between the benchmarks in this experiment is in their task graph structure. Also note that the since the tasks are computed immediately, any potential attempts to steal a task from a worker will fail. Nonetheless, the workers will still respect the initial assignments from the scheduler.

In addition to makespan, we evaluate the average runtime overhead per task (AOT), which is calculated as the makespan divided by the number of tasks in the task graph. Figure 6 shows speedup of RSDS against DASK when the zero worker is used. Results shows that RSDS is $1.1-6 \times$ faster. This is a larger difference than when the standard worker was used, which suggests that RSDS would benefit from a faster worker implementation more than the DASK server.

The DASK manual states that "Each task suffers about $1 \mathrm{~ms}$ of overhead. A small computation and a network roundtrip can complete in less than 10ms." 10 Our tests with AOT show that the overhead is less than $1 \mathrm{~ms}$ for most of our benchmarks. Results are shown in Figure 7

Figure 8 shows AOT for the merge benchmark. The top graph shows how AOT changes when the number of tasks is increased, the bottom graph shows how AOT changes with an increasing number of workers.

\footnotetext{
${ }^{10}$ https://distributed.dask.org/en/latest
}

The results show that the random scheduler has less overhead and its overhead does not increase as fast with an added number of workers as with the work-stealing scheduler, which is expected. This effect is also confirmed by the bottom chart of Figure 8, where the overhead of the random scheduler stays almost constant when more workers are added.

This data indicates that the general runtime overhead of DASK mainly grows with an increasing number of tasks, no matter which scheduler is used. On the other hand, overhead of the work-stealing scheduler grows primarily with the number of workers. In the case of RSDS, work-stealing overhead stays constant for up to 100 workers, then it also starts to grow with additional workers. It also shows an increasing tendency when the number of tasks is increased. However, its overhead stays well under DASK on all benchmarked configurations.

\section{CONCLUSION}

In our paper we have studied the performance of DASK, a distributed task framework with a central scheduler. We have demonstrated that the benefits of a sophisticated scheduler design might be dwarfed by runtime inefficiencies of the task framework - in other words, unless the runtime is properly optimized first, it might not be worthwhile to spend too much time on clever scheduler designs. We show that for some task graphs even a completely random scheduler is competitive with the DASK built-in scheduler, especially with larger clusters. Our experiment with an idealized worker implementation has quantified the per-task overhead of DASK. Our experiments demonstrate that for an embarrassingly parallel benchmark, the overhead of the DASK runtime grows with the number of tasks, independently on the used scheduler. On the other hand, the performance of the work-stealing scheduler is mainly affected by the amount of workers in the cluster.

We have introduced RSDS, a drop-in replacement of the DASK server that outperforms the original server implementation even though it uses a simpler and less tuned scheduler. RSDS is open-source and can be used to accelerate common existing DASK workflows without any source code changes.

In future work, we would like to explore the effects of network topology and bandwidth on the performance of the DASK scheduler. It would also be interesting to quantify the effect of improving worker performance on the overall workflow runtime.

\section{REFERENCES}

[1] M. Rocklin, "Dask: Parallel computation with blocked algorithms and task scheduling." Citeseer, 2015.

[2] P. Moritz, R. Nishihara, S. Wang, A. Tumanov, R. Liaw, E. Liang, M. Elibol, Z. Yang, W. Paul, M. I. Jordan et al., "Ray: A distributed framework for emerging $\{\mathrm{AI}\}$ applications," in 13th $\{$ USENIX $\}$ Symposium on Operating Systems Design and Implementation ( $\{O S D I\} 18)$, 2018.

[3] M. Zaharia, M. Chowdhury, M. J. Franklin, S. Shenker, and I. Stoica, "Spark: Cluster computing with working sets," in Proceedings of the 2nd USENIX Conference on Hot Topics in Cloud Computing, ser. HotCloud'10. USA: USENIX Association, 2010.

[4] J. D. Ullman, "NP-complete scheduling problems," J. Comput. Syst. Sci., vol. 10 , no. 3, Jun. 1975 

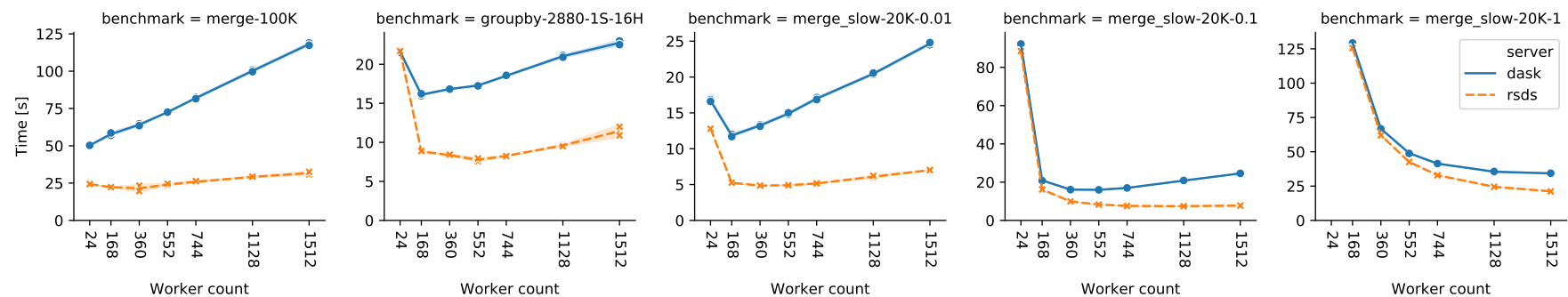

Fig. 5. Strong scaling of DASK and RSDS on selected task graphs

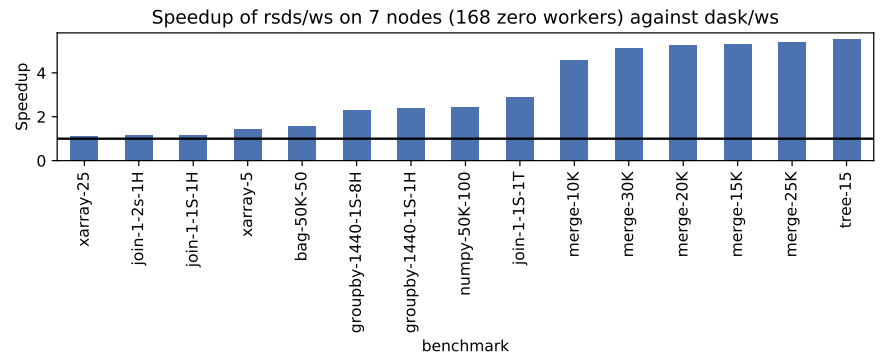

Fig. 6. Speedup of RSDS/ws over DASK/ws when zero worker is used

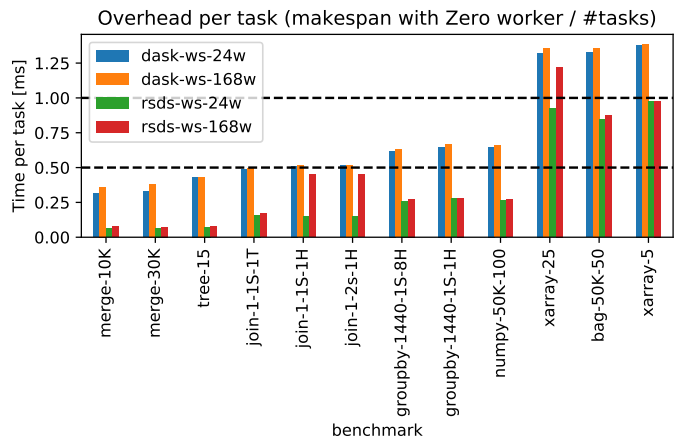

Fig. 7. Overhead per task for various cluster sizes and benchmarks
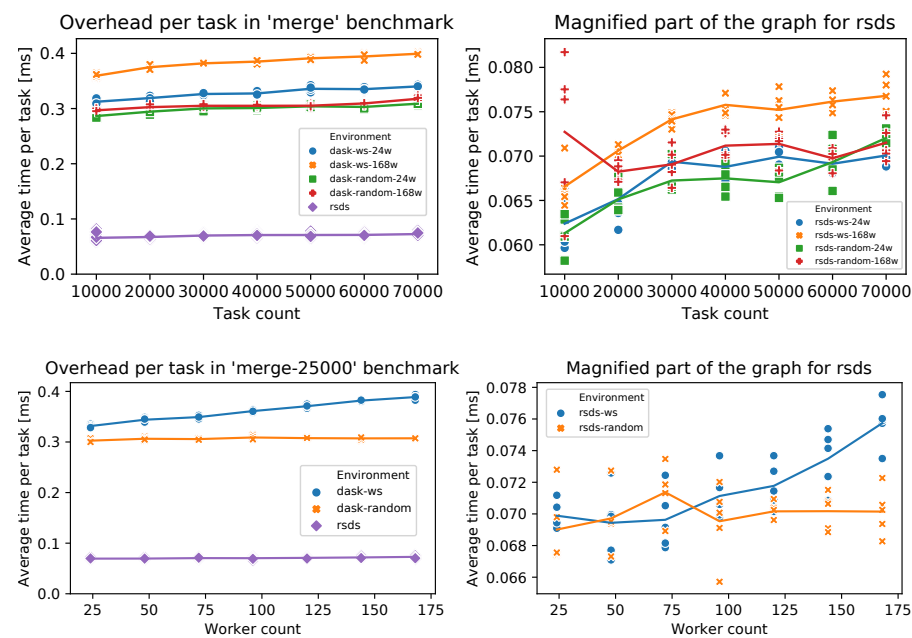

Fig. 8. Average task times in the merge benchmark when zero worker is used: (top) scaling task count; (bottom) scaling worker count.
[5] G. C. Sih and E. A. Lee, "A compile-time scheduling heuristic for interconnection-constrained heterogeneous processor architectures," IEEE transactions on Parallel and Distributed systems, vol. 4, no. 2, 1993.

[6] M.-Y. Wu and D. D. Gajski, "Hypertool: A programming aid for messagepassing systems," IEEE transactions on parallel and distributed systems, vol. 1 , no. 3, 1990

[7] T. L. Adam, K. M. Chandy, and J. R. Dickson, "A comparison of list schedules for parallel processing systems," Commun. ACM, vol. 17, no. 12, Dec. 1974.

[8] Y.-K. Kwok and I. Ahmad, "Static scheduling algorithms for allocating directed task graphs to multiprocessors," ACM Computing Surveys (CSUR), vol. 31, no. 4, 1999

[9] X. Tang, K. Li, G. Liao, and R. Li, "List scheduling with duplication for heterogeneous computing systems," Journal of parallel and distributed computing, vol. 70, no. 4, 2010.

[10] M. I. Daoud and N. Kharma, "A hybrid heuristic-genetic algorithm for task scheduling in heterogeneous processor networks," Journal of Parallel and Distributed Computing, vol. 71, no. 11, 2011.

[11] E. Popa, M. Iacono, and F. Pop, "Adapting mep and hlfet algorithms to multiple simultaneous scheduling," International Journal of Parallel Programming, vol. 46, no. 3, 2018.

[12] Y.-K. Kwok and I. Ahmad, "Benchmarking the task graph scheduling algorithms," in ipps. IEEE, 1998.

[13] T. Hagras and J. Janeček, "Static vs. dynamic list-scheduling performance comparison," Acta Polytechnica, vol. 43, no. 6, 2003.

[14] H. Wang and O. Sinnen, "List-scheduling vs. cluster-scheduling," IEEE Transactions on Parallel and Distributed Systems, 2018.

[15] J. Beránek, S. Böhm, and V. Cima, "ESTEE: A simulation toolkit for distributed workflow execution (poster abstract)," The International Conference for High Performance Computing, Networking, Storage, and Analysis 2019.

[16] N. D. Matsakis and F. S. Klock, "The Rust language," in Proceedings of the 2014 ACM SIGAda Annual Conference on High Integrity Language Technology, ser. HILT '14. New York, NY, USA: Association for Computing Machinery, 2014.

[17] M. Rocklin. Dask benchmarks. [Online]. Available: https: //matthewrocklin.com/blog/work/2017/07/03/scaling

[18] E. Slaughter, W. Wu, Y. Fu, L. Brandenburg, N. Garcia, W. Kautz, E. Marx, K. S. Morris, W. Lee, Q. Cao et al., "Task Bench: A parameterized benchmark for evaluating parallel runtime performance," arXiv preprint arXiv:1908.05790, 2019.

[19] M. Dugré, V. Hayot-Sasson, and T. Glatard, "A performance comparison of dask and apache spark for data-intensive neuroimaging pipelines," in 2019 IEEE/ACM Workflows in Support of Large-Scale Science (WORKS). IEEE, 2019.

[20] A. Watson, D. S. V. Babu, and S. Ray, "Sanzu: A data science benchmark," in 2017 IEEE International Conference on Big Data (Big Data). IEEE, 2017.

[21] V. Cima, S. Böhm, J. Martinovič, J. Dvorskỳ, K. Janurová, T. V. Aa, T. J. Ashby, and V. Chupakhin, "Hyperloom: a platform for defining and executing scientific pipelines in distributed environments," in PARMADITAM@HiPEAC'18, 2018.

[22] K. et al., "The ncep/ncar 40-year reanalysis project," Bull. Amer. Meteor. Soc., 77, 437-470, 1996.

[23] Benchmarking python distributed AI backends with wordbatch. [Online]. Available: https://towardsdatascience.com/ benchmarking-python-distributed-ai-backends-with-wordbatch-9872457b785c 